当 $\boldsymbol{Z}=\left(Z_{1}, \cdots, Z_{n}\right) \in \mathscr{F}_{n}$ (即 $\mathscr{L}(\boldsymbol{Z}) \in \mathscr{F}_{n}$ ) 时, 概率 $P\left(Z_{1}>z_{1}, \cdots, Z_{n}>z_{n}\right)$ 是 $\Sigma_{1}^{n} z_{k}$ 的函数, 故又可 定义另一分布族

$$
\begin{aligned}
\mathscr{J}_{n} & =\left\{\mathscr{L}(\boldsymbol{Z}): \boldsymbol{Z} \in R_{+}^{n}, P\left(Z_{1}>z_{1}, \cdots, Z_{n}>z_{n}\right)\right. \\
& =h\left(\Sigma_{1}^{n} z_{k}\right)
\end{aligned}
$$

对任 $\left(z_{1}, \cdots, z_{n}\right) \in R_{+}^{n}$ 及 $[0, \infty)$ 上的函数 $\left.h(\cdot)\right\}$ 若 $\mathscr{L}(\boldsymbol{Z}) \in \mathscr{T}_{n}$ 且

$$
P\left(Z_{1}>z_{1}, \cdots, Z_{n}>z_{n}\right)=h\left(\Sigma_{1}^{n} z_{k}\right),
$$

记为 $\boldsymbol{Z} \in \mathscr{T}_{n}(h)$. 我们又得到如下结果:

1. 设 $\boldsymbol{Z} \in R_{+}^{n}, P(\boldsymbol{Z}=\mathbf{0})=0, \boldsymbol{z}$ 有密度, 则下 列条件等价:

(1) $\boldsymbol{Z} \in \mathscr{F}_{n}(G), G^{\prime}(r)=g(r) \geqslant 0$, a.e., $r \geqslant 0$.

(2) $\boldsymbol{Z} \in \mathscr{T}_{n}(h), h^{(n)}(t)$ 存在, 不 a.e. 为 $0, t$ $\geqslant 0$.

（3） Z 有密度形如 $f\left(\sum_{k=1}^{n} z_{k}\right),\left(z_{1}, \cdots z_{n}\right) \in R_{+}^{n}$ 此时有

$$
\begin{gathered}
\Gamma(n) x^{-n+1} g(x)=f(x)=(-1)^{n} h^{(n)}(x), x>0, \\
h(t)=\Gamma(n)^{-1} \int_{0}^{\infty} f(x+t) x^{n-1} d x, \\
t \geqslant 0 .
\end{gathered}
$$

2. 设 $Z \in R_{+}^{n}, n \geqslant 2$, 则下列条件等价:
(1) $\boldsymbol{Z} \in \mathscr{F}_{n}(G), G(0)=0$.

(2) $Z \in \mathscr{T}_{n}(h), h(0)=1$ 且 $h$ 为 $(0, \infty)$ 上的 $n$ 次单调函数.

此时有

$$
h(t)=\int_{(t, \infty)}(1-t / r)^{n \cdot 1} d G(r), t \geqslant 0 .
$$

3. 设 $\boldsymbol{Z} \in R_{+}^{n}, G(\cdot)$ 为 $(0, \infty)$ 上的分有涵数, 则下列条件等价:

(1) $\boldsymbol{Z} \in \bigcap_{k=1}^{\infty} D_{n, n+k}$ 且 $P\left(Z_{\mathrm{t}}=0\right)=0$, 其中

$D_{n, n+k}=\left\{\mathscr{L}(\boldsymbol{Z}): \boldsymbol{Z} \in R_{+}^{n}\right.$ 且存在 $\boldsymbol{W} \in K_{+}^{k}$, 使 $\left.(\boldsymbol{Z}, \boldsymbol{W}) \in \mathscr{F}_{n+k}\right\}$.

(2) $\boldsymbol{Z} \in \mathscr{T}_{n}(h)$ ，其中

$$
h(t)=\int_{(0, \infty)} e^{\cdots t / r} d G(r), t \geqslant 0 .
$$

（3） Z有密度 $f\left(\sum_{k=1}^{n} z_{k}\right),\left(z_{1}, \cdots, z_{n}\right) \in R_{+}^{n}$, 其中

$$
f(x)=\int_{(0, \infty)} e^{-x / r_{r}-n} d G(r), x>0 .
$$

(4) $\boldsymbol{Z}=R \boldsymbol{X} \in D_{\boldsymbol{n}, \infty}$

本文还给出了若干应用.

方开泰 方碧琪

(中国科学院应用数学研究所, 北京)

\title{
稳定自由基配合剂及其配合物的研究*
}

稳定自由基配合剂分子中带有能键合金属离子 的配合基团,能与顺磁性、反磁性金属离子配合,所 形成的配合物又保留原试剂的自由基特性，从而根 据配合物的 ESR 谱线形状和强度，可了解配合物的 结构, 并推求其浓度. 它可以作为检测金属离子的 高灵敏度方法。

我们合成了 4 -黄原酸钾 $-2,2,6,6$ - 四甲基哌 啶-1-氧基化合物（KA)**，4-羧基-4-胺基-2，2， 6,6-四甲基哌啶-1-氧基化合物 $(\mathrm{HB})^{* *}$ 及其 $\mathrm{C}_{2} 、 \mathrm{Ni}$ 、 $\mathrm{Zn}$ 的配合物, 并研究了它们的结构.

黄原酸系配合物的 $\mathrm{xp}$ 结果表明黄原酸基的硫 与金属离子发生了配位, 且黄原酸铜中的铜为二价。 配合物 $\mathrm{NiA}_{2}$ 的 ESR 谱为三重峰，与 $\mathrm{KA}$ 相同; $\mathrm{CuA}_{2}(\mathrm{OH})_{2}$ 与黄原酸二聚物 $\mathrm{A}_{2}$ 相同, 为五重峰, 且 在四氯化碳、三氯甲烷、四氢呋喃、丙酮和苯的溶 液中, 都显示五重峰, 溶剂效应不明显. 红外和紫 外吸收光谱以及差热分析结果也都表明 $\mathrm{NiA}_{2}$ 和 $\mathrm{CuA}_{2}(\mathrm{OH})_{2}$ 的性质分别与 $\mathrm{KA}$ 和 $A_{2}$ 相近. 可设想
$\mathrm{NiA}_{2}$ 与 $\mathrm{CuA}_{2}(\mathrm{OH})_{2}$ 的结构分别为:<smiles>CC12[Al]C(C)(S1)C(OC1CC(C)(C)C(C)(C)C1)S2</smiles>

$(\mathrm{OH})_{2}$<smiles>COC1CC(C)(C)N([O])C1(C)C</smiles>

HB 系配合物难溶于各种极性与非极性溶剂中, 它在四氢呋喃中的 ESR 谱显示三重峰. xps 结果表 明配位原子是羧基氧而不是胺基氮. 其结构可能为:

* 中国科学院科学基金资助的课题.

**A、B 分别为配合剂的阴离子. 


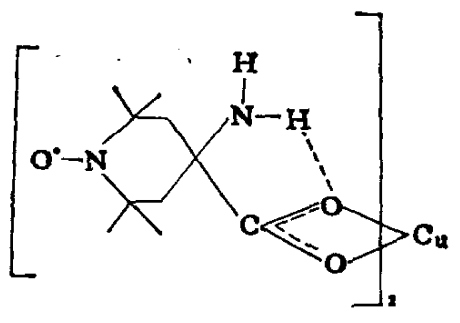

和邻苯二甲酸与 $\mathrm{Cu}$ (II) 的配合物的结构相似. 招䘵基 高锦春 笪有仙 徐广智 (中国科学院化学研究所, 北京)

\section{铁 (III)-邻二氮菲溶液的光化学还原}

含乙酸、柠檬酸等有机酸或乙酰丙酮、8-羟基 喹啉等有机配体的铁 (III)-邻二氮菲 (phen) 溶液, 能发生光化学还原反应, 生成 $\left[\mathrm{Fe}(\mathrm{phen})_{3}\right]^{2+}$. 该现 象至今仍被忽视，或被简单归结为 Wehry 和 David 等人曾研究过的 $[\mathrm{Fe}(\mathrm{phen})]^{3+}$ 和双核配离子

$$
\left[(\mathrm{phen})_{2}\left(\mathrm{H}_{2} \mathrm{O}\right) \mathrm{Fe}-\mathrm{O}-\mathrm{Fe}\left(\mathrm{H}_{2} \mathrm{O}\right)(\mathrm{phen})_{2}\right]^{4+}
$$

在溶液中的光化学还原. 或如 Hey 所认为的, 在溶 液中发生了某种氧化还原反应。我们吕 $\mathrm{pH} \mathrm{s}-2$ 型 酸度计、WDF 型反射式单色光计、721 型分光光度 计和气相色谱仪、以 GD30 紫外灯、300W 高压铟灯、 $110 \mathrm{~W}$ 高压钠灯、GGY-125W 苂光高压永灯、太阳光 等为光源, 对 $\mathrm{Fe}^{3+}$-phen 溶液体系进行了光化学还 原研究, 发现只有当 $\mathrm{pH}$ 值大于 $1.0-1.5$ (与 $\mathrm{Fe}^{3+}$ 浓 度有关) 时, 溶液体系才能发生光化学还原反应. 不含其它有机配体时，光化作用的有效波长 $<300$ $\mathrm{nm}$; 含乙酸时, 为 $<300 \mathrm{~nm}$ 和在 $420-450 \mathrm{~nm}$; 含 柠檬酸时, 为 $420-450 \mathrm{~nm}$ 。苂光高压录灯、太阳光、 高压铟灯等是该光化反应的有效光源. 当有过量 phen 和其它有机配体存在时, 含 $\mathrm{Fe}^{3+} 1 \sim 10 \mu \mathrm{g} / \mathrm{ml}$ 的溶液在几分钟到半小时内, $\mathrm{Fe}^{3+}$ 已完全转化为 $\left[\mathrm{Fe}(\mathrm{phen})_{3}\right]^{2+} . \mathrm{F}^{-}, \mathrm{Cl}^{-}, \mathrm{PO}_{4}^{3-}, \mathrm{OH}^{-}$( $\mathrm{pH}>10$ 时)
等与 $\mathrm{Fe}^{3+}$ 配位的无机离子, 严重抑制光化还原反 应. 体系中不含其它有机配体时, 光化反应中有 $\mathrm{O}_{2}$ 产生, 表明水为电子供体:

$$
\begin{gathered}
\mathrm{H}_{2} \mathrm{O} \rightleftharpoons \mathrm{H}^{+}+\mathrm{OH}^{-}, \\
\mathrm{Fe}(\mathrm{OH})^{2+} \stackrel{h \nu}{\longrightarrow} \mathrm{Fe}^{2+}+\cdot \mathrm{OH}^{2} \\
2 \cdot \mathrm{OH} \longrightarrow \mathrm{H}_{2} \mathrm{O}+\frac{1}{2} \mathrm{O}_{2} .
\end{gathered}
$$

体系中含羧酸等有机配体时，光化反应中有 $\mathrm{CO}_{2}$ 产生, 表明有机配体为电子供体, 以羒酸为例:

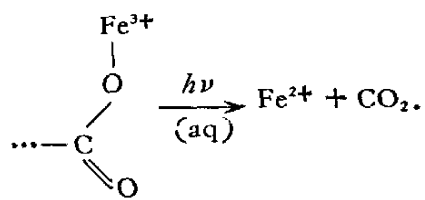

该研究结果对改进传统的 phen 测铁法具有重 要价值. 我们已研究成功用 phen 连续测定同一份 试液中 $\mathrm{Fe}^{2+}$ 和 $\mathrm{Fe}^{3+}$ 含量的分光光度一光化学还原 法, 从而, 解决了英国化学家 Hey 曾提出的这一分 析化学上的难题.

颜 科 沙德仁 谭明霞 (南京玻璃纤维研究设计院)

\section{电镀法制取铁磷非晶态合金}

我们对用电镀方法制取非晶态新材料进行了研 究, 制得了铁磷非晶态合金镀层。

此合金含磷量为 $7-5 \%$, 是在硫酸亚铁镀液中 加一定的添加剂配制而成的. 根据需要加入其它添 加剂, 还可镀成多元合金.

锫层主要性能如下:

1. 硬度: HRC $45 \sim 60 ; 400 \sim 500^{\circ} \mathrm{C}$ 加热一小 时后硬度可升至 $900-1,400 \mathrm{HV}_{0.1}$.
2.耐磨性能: 优于铁镇、铁镍及纯铁镀层, 磨痕 宽度依次为: $2.58 ; 2.57 ; 2.77(\mathrm{~mm})$ 而铁磷非晶镀 层为 $1.83 \mathrm{~mm}$.

3. 用 X 射线衍射法测定镀层结构、为典型的非 晶弥散峰.

4.金相观察: 用 $4 \%$ 硝酸酒精浸蚀, 放大 $400 \times$, 光学显微镜下观察不到任何组织和裂纹.

5 . 磁性: 厚 $0.035 \mathrm{~mm}$ 均匀截面积为 $0.2 \mathrm{~mm}^{2}$ 